\title{
Self-propelling, coalescing droplets
}

\author{
M. Sellier* \\ Department of Mechanical Engineering, The University of Canterbury, \\ Private Bag 4800, Christchurch 8140, New Zealand \\ V. Nock \\ Department of Electrical and Computer Engineering, \\ The University of Canterbury, Private Bag 4800, Christchurch 8140, New Zealand

$$
\text { C. Verdier }{ }^{\ddagger}
$$ \\ Laboratoire de Spectrométrie Physique, \\ CNRS and Université Grenoble I, UMR 5588, \\ 140 Avenue de la Physique, BP 8\%, \\ 38402 Saint-Martin d'Hères, France
}




\begin{abstract}
This work proposes and explores a new propulsion mechanism for sessile droplets which could be of interest for microfluidic applications. This mechanism relies on the Marangoni stresses resulting from the surface tension gradient arising when two droplets of different surface tensions coalesce. We argue that the tendency of the fluid to flow towards regions of higher surface tension is sufficient to displace the droplet. The coalescence of two miscible, partially wetting droplets with different surface tensions is investigated theoretically in this paper and modeled in the lubrication approximation framework. The problem is described by a set of three highly non-linear, coupled partial differential equations which is solved with a commercial finite element code. The analysis reveals two important dimensionless numbers which govern the flow characteristics, one related to the strength of the surface tension gradient and the other to the diffusion time scale. The numerical results confirm the occurrence of the self-propulsion behavior and a parametric study is performed to explore the role of the two dimensionless numbers on the propulsion speed and the total displacement. Unsurprisingly, self-propulsion is enhanced for larger values of the surface tension contrast between the two droplets and smaller values of the diffusion time scale which results in more time for the surface tension gradient to act.
\end{abstract}

\footnotetext{
*mathieu.sellier@canterbury.ac.nz; http://www.mech.canterbury.ac.nz/people/sellier.shtml

$\dagger$ volker.nock@canterbury.ac.nz

‡verdier@ujf-grenoble.fr
} 


\section{INTRODUCTION}

Microfluidic devices play an ever increasing role in nano- and biotechnologies. An emerging area of research in this technology-driven field is digital microfluidics which is based upon the micromanipulation of discrete droplets (Fair et al., 2007; Darhuber and Troian, 2005). Microfluidic processing is performed on unit-sized packets of fluid which are transported, stored, mixed, reacted, or analyzed in a discrete manner. An obvious challenge however is how to displace the sessile droplets on the substrate. A range of propulsion mechanisms has been investigated in the past including chemically treating the substrate such that the wettability becomes non-uniform (see e.g. Greenspan 1978; Brochard 1989; Chaudhury and Whitesides 1992; Daniel and Chaudhury 2002), applying an electrical potential to exploit the electrowetting effect, (see e.g. Cho et al. 2003; Yeo and Chang 2005; Cho and Moon 2008), applying surface acoustic waves, (see e.g Renaudin et al. 2006; Yeo and Friend 2009), or inducing a gradient of surface tension by applying a temperature gradient, the so-called Marangoni effect, (see e.g Brzoska et al 1993; Darhuber et al 2003a; Darhuber et al 2003b; Ford and Nadim 1994; Smith 1995; Yarin et al. 2002; Gomba and Homsy 2010).

This work investigates the possibility to propel the droplets using the surface tension gradient which arises during the mixing of two droplets of liquid having different compositions and therefore surface tensions. The fluid having a tendency to flow towards regions of higher surface tension, a net flow results and one would expect the droplets to move until a fully mixed state is reached resulting in a uniform surface tension or "fuel" exhaustion. This problem is treated theoretically here to demonstrate the principle and builds on earlier work on the modeling of the coalescence of sessile droplets (see Sellier and Trelluyer 2009). The coalescence of sessile droplets is a problem which has recently attracted the attention of researchers because of their relevance in coating phenomena such as spray coating, liquid and chemical imbibition on plant foliage, or in microfluidic devices for which the coalescence of sessile droplets has recently been identified as a potential mechanism to mix reagents (see e.g. Ristenpart et al. 2006 or Stone et al. 2004). It is explored here for the first time as a possible propulsion mechanism.

The next section is devoted to the description of the problem, the model, and the solution procedure. It is followed by a result section which demonstrates the self-propulsion mechanism and investigate the influence of important dimensionless parameters. The paper 
closes on concluding remarks.

\section{MODEL DESCRIPTION}

\section{A. Geometry and notations}

The problem considered is illustrated in Figure 1. Two out of equilibrium droplets (Droplet 1 and Droplet 2) having the same viscosity $\mu$ and density $\rho$ spread independently and contact at time $T_{0}$ when coalescence begins. The miscible, non-volatile droplets have the same initial volume $V_{0}$ and they partially wet the substrate forming a characteristic contact angle $\Theta_{s}$ at equilibrium. Droplet 1 is initially centered at $\left(X_{1}, Y_{1}\right)$ and Droplet 2 at $\left(X_{2}, Y_{2}\right)$. The surface tensions of Droplet 1 and 2 are $\sigma_{1}$ and $\sigma_{2}$, respectively. The Marangoni stresses arising from the surface tension gradient induced during the mixing are expected to produce a net flow in the direction of increasing surface tension as shown in Figure 1. We assume, as done in Schwartz et al. (1995) or Howison et al. (1997), that the surface tension of the mixture of Droplets 1 and 2 is simply given by

$$
\sigma=\sigma_{1}+\Gamma(1-c)
$$

where $\Gamma=\sigma_{2}-\sigma_{1}$ and $c$ is the local concentration of Droplet 1 fluid.

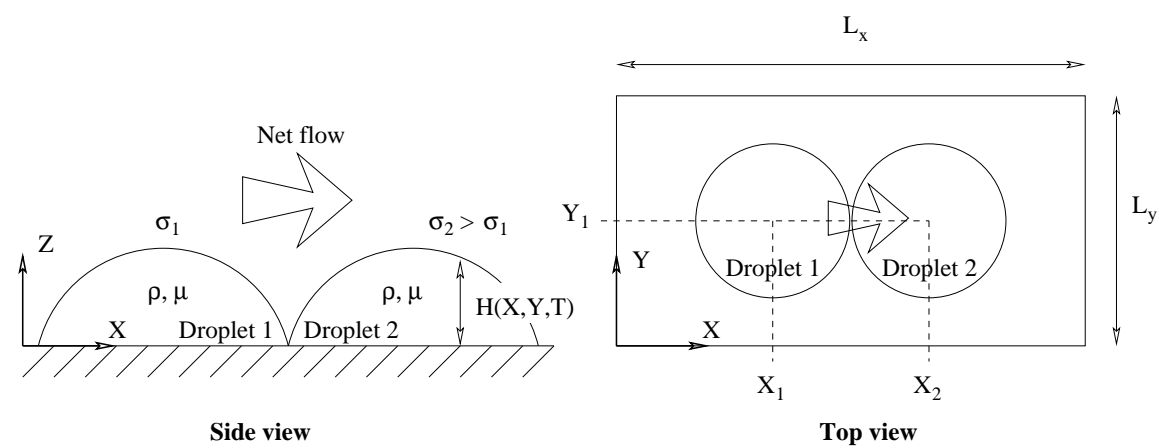

FIG. 1. Sketch of the droplets system at the onset of coalescence.

\section{B. Governing equations}

Like most free boundary problems, modeling the coalescence of sessile droplets is challenging and the presence of a dynamic wetting line is an additional source of complexity 
because of the ill-defined nature of the boundary condition along this line. The present study builds on the model described by Sellier and Trelluyer (2009) where this free surface problem is tackled in the lubrication approximation framework and a disjoining pressure model is used to capture the dynamics of the wetting front. The main idea, described in details in other sources (see de Gennes 1985; Schwartz and Eley 1998; Schwartz 1998), is to introduce a thin precursor film of thickness $H^{\star}$ ahead of the contact line to remove the singularity there and add a disjoining pressure term of the correct magnitude such that the motion of the contact line ceases when the contact angle reaches its equilibrium value. In spite of its limitations, such modeling framework has had some success in predicting the dynamics of droplet spreading on homogeneous or heterogeneous substrates (see e.g. Schwartz and Eley 1998; Schwartz 1998; Gaskell et al. 2004). The resulting governing equations read

$$
\begin{aligned}
\frac{\partial H}{\partial T} & =-\nabla \cdot \vec{Q} \\
\frac{\partial c H}{\partial T} & =-\nabla \cdot(c \vec{Q})+D \nabla \cdot(H \nabla c), \\
\vec{Q} & =-\left(\frac{H^{3}}{3 \mu} \nabla P\right)+\left(\frac{H^{2}}{2 \mu} \nabla \sigma\right), \\
P & =-\sigma \nabla^{2} H-\Pi\left(H, H^{\star}\right)
\end{aligned}
$$

where the droplet thickness normal to the substrate $H(X, Y, T)$, the concentration $c(X, Y, T)$ of Droplet 1 phase, and the pressure $P(X, Y, T)$ are the dependent variables and $D$ is the diffusivity constant. Equation (2) expresses the conservation of mass and eq. (5) the balance of normal stresses at the interface. The discharge $\vec{Q}$, given by eq. (4), has two contributions: the first one results from the pressure gradient and the second one from the surface tension gradient (Marangoni stresses). Equation 3 is a "depth-averaged" statement of the conservation of Droplet 1 phase in the mixture derived in a similar form by Howison et al. (1997). The disjoining pressure term is given by

$$
\Pi\left(H, H^{\star}\right)=\frac{(n-1)(m-1)}{H^{\star}(n-m)} \sigma\left(1-\cos \Theta_{s}\right)\left[\left(\frac{H^{\star}}{H}\right)^{n}-\left(\frac{H^{\star}}{H}\right)^{m}\right],
$$

where $n$ and $m$ are the exponents of the interaction potential. Note that the disjoining pressure term is zero for a fully wetting system since $\Theta_{s}=0$ for such systems. Notwithstanding corrections in the contact line region, a steady solution of eqs. (2) and (5) in the absence of surface tension gradient is a paraboloid of revolution. Based on the equilibrium configuration of a droplet of volume $V_{0}$ lying on a substrate with equilibrium contact angle 
$\Theta_{s}$, a characteristic droplet radius $R_{0}$ and maximum thickness $H_{0}$ can be defined. Indeed, if we assume the equilibrium droplet to be a paraboloid of revolution, its volume will be $V_{0}=\frac{\pi}{2} H_{0} R_{0}^{2}$ and its contact angle $\Theta_{s}=\arctan \left(\frac{2 H_{0}}{R_{0}}\right)$ or $\Theta_{s} \approx \frac{2 H_{0}}{R_{0}}$ in the limit of small $\epsilon=\frac{H_{0}}{R_{0}}$ relevant to the lubrication approximation. The latter can be rearranged to express the droplet radius and thickness as

$$
\begin{aligned}
& R_{0}=\sqrt[3]{\frac{4 V_{0}}{\pi \Theta_{s}}}, \\
& H_{0}=\frac{\Theta_{s}}{2} \sqrt[3]{\frac{4 V_{0}}{\pi \Theta_{s}}} .
\end{aligned}
$$

Introducing these scales along with $T_{0}=\frac{3 \mu R_{0}^{4}}{\sigma H_{0}^{3}}$ in the governing equations results in

$$
\begin{aligned}
\frac{\partial h}{\partial t} & =\nabla \cdot\left(-\Sigma h_{1} \nabla h+\Sigma h \nabla h_{1}+h^{3} \nabla p\right), \\
\frac{\partial h_{1}}{\partial t} & =\nabla \cdot\left(\left(-\Sigma \frac{h_{1}^{2}}{h}-d \frac{h_{1}}{h}\right) \nabla h+\left(\Sigma h_{1}+d\right) \nabla h_{1}+h_{1} h^{2} \nabla p\right), \\
p & =-\nabla^{2} h-B\left[\left(\frac{h^{\star}}{h}\right)^{n}-\left(\frac{h^{\star}}{h}\right)^{m}\right],
\end{aligned}
$$

where $\Sigma=\frac{3 \Gamma}{2 \sigma_{1} \epsilon^{2}}, d=\frac{D T_{0}}{R_{0}{ }^{2}}, h^{\star}$ is the dimensionless precursor film thickness and $B=$ $2 \frac{(n-1)(m-1)}{h^{\star}(n-m)}$. The concentration $c$ of Droplet 1 phase is replaced by the fictitious "Droplet 1 height" $h_{1}=c h$. The constants $(n, m)$ are set to $(9,3)$ which corresponds to a Lennard-Jones interaction potential (Schwartz and Eley 1998). The pressure $P$ is scaled with $P_{0}=\frac{\sigma \epsilon}{R_{0}}$. The time scale introduced here is the one used by Sellier and Trelluyer in [24] to describe the coalescence dynamics of identical sessile droplets and generally speaking, it is the time scale relevant to capillary induced flows. It can easily be obtained by noting that a characteristic velocity for thin layer flows driven by surface tension is $V_{C}=R_{0} / T_{0} \approx H^{2} \nabla P / 3 \mu$ with $P=-\sigma \nabla^{2} H$ from which the characteristic time scale $T_{0}=\frac{3 \mu R_{0}^{4}}{\sigma H_{0}^{3}}$ can easily be recovered. In the present case, it is worth noting that there are two complementary driving mechanisms: the capillary and Marangoni effects. Both phenomena have their own intrinsic time scales and the subsequent results show that, typically, they are not the same. Capillary acts on a short time scale to merge the two droplets together whilst surface tension gradients act on a longer time scale to displace the merged droplets system. For reasons of consistency with our earlier work, we use here the time scale representative of capillary induced flows. Notwithstanding the variable surface tension effects, this model is somewhat related to the one proposed (but not implemented) by Andrieu et al. (2002) although they use a different approach to alleviate the singularity at the contact line. 


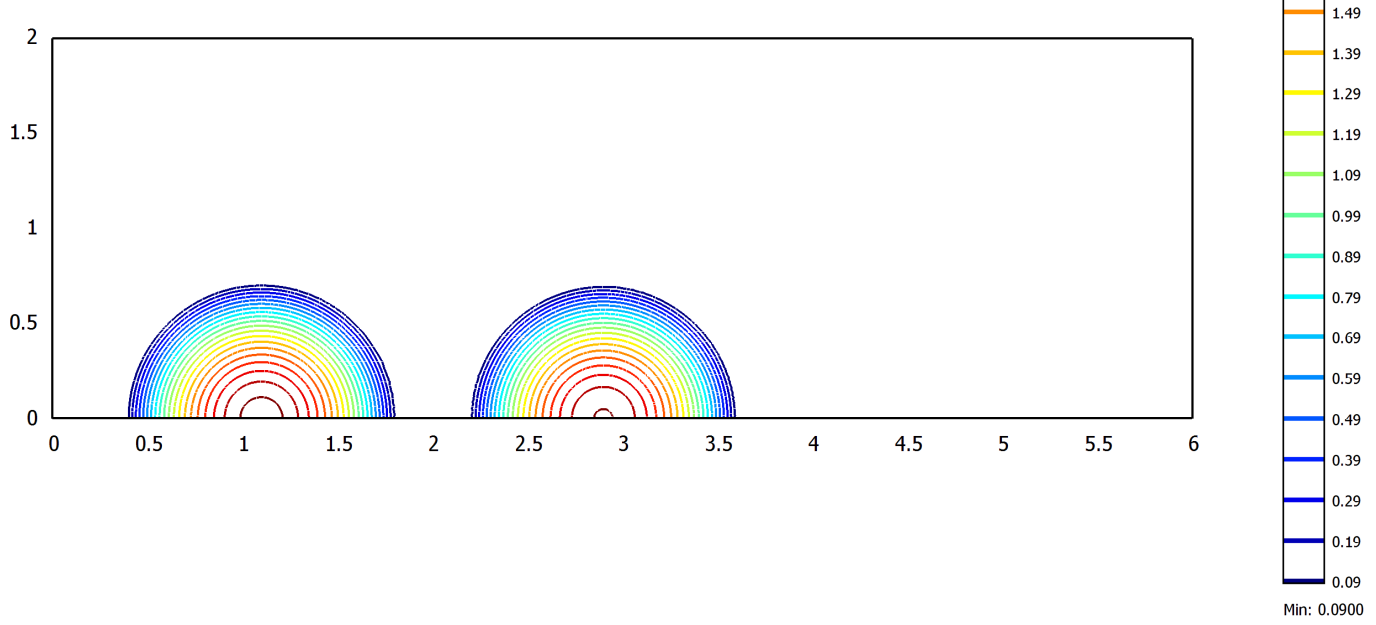

FIG. 2. Computational domain with the initial droplet locations. The droplets are seen from above and only half of the droplets system is modeled here for symmetry reasons. The lines are contours of equal droplet height.

\section{Solution procedure}

The governing equations are solved in the commercial Finite Element package COMSOL. This package is particularly convenient for solving arbitrary partial differential equations. Details on the implementation can be found in Sellier and Panda (2010). Since the line joining the centers of the droplets is a symmetry line, only half of the domain needs to be considered, as shown in Figure 2. The computational domain is a $L_{x} \times L_{y}=6 R_{0} \times 2 R_{0}$ rectangle and the line $y=0$ correspond to the symmetry line. The computational domain is tesselated with approximately 19,000 quadratic, Lagrange triangular elements. Given our model and scales, a paraboloid of radius and height equal to unity is the equilibrium configuration for a droplet of volume $\frac{\pi}{2} H_{0} R_{0}{ }^{2}$ (dimensional) or $\frac{\pi}{2}$ (dimensionless). As an initial condition to the coalescence problem, we choose two droplets out of equilibrium with initial height $2 H_{0}$ (or 2 in dimensionless units) and volume $\frac{\pi}{2} H_{0} R_{0}{ }^{2}$, the centroids of which 
are placed $1.8 R_{0}$ apart such that the coalescence occurs when the droplets have almost reached their maximum spread radius. The initial profile for each of the droplets ( 1 and 2) is $h_{\text {ini }}(r)=\max \left[h^{\star}, h^{\star}+2\left(1-2 r^{2}\right)\right]$, where $r$ is the distance from the centroid of each droplet located at $(1.1,0)$ and $(2.9,0)$ in dimensionless units. The "Droplet 1 height" $h_{1}$ is set to $h_{i n i}$ inside Droplet 1 resulting in a unit concentration there and to zero everywhere else. Natural boundary conditions are applied at the edge of the computational domain such that $\nabla h \cdot \vec{n}=\nabla p \cdot \vec{n}=\nabla h_{1} \cdot \vec{n}=0$ where $\vec{n}$ is the normal to the boundary pointing outward. Since the droplets remain in the computational domain at all time, the solution appears to be insensitive to the latter.

\section{RESULTS AND DISCUSSION}

The governing equations, eqs. (9), (10), and (11), show that the problem is essentially governed by two dimensionless parameters, i.e. $\Sigma$ which is a measure of the strength of the Marangoni stresses and $d$, the diffusivity. Before proceeding any further, a rough assessment of the order of magnitude of the different parameters is necessary. As a possible scenario, let's assume that the droplets have a volume of $10 \mu \mathrm{l}$ and a static contact angle of $40^{\circ}=0.7 \mathrm{rad}$. The corresponding equilibrium droplet radius and height, calculated according to eqs. (7) and (8), are $R_{0}=2.62 \mathrm{~mm}$ and $H_{0}=1.19 \mathrm{~mm}$, respectively. If the fluid considered is silicone oil with surface tension $\sigma_{1}=0.02 \mathrm{~N} / \mathrm{m}$ and viscosity $\mu=0.0965 \mathrm{~N} / \mathrm{m}$, the corresponding time scale $T_{0}$ is $0.4 \mathrm{~s}$. For a surface tension contrast $\Gamma$ of $0.01 \mathrm{~Pa}$.m, this results in $\Sigma$ being equal to 3.64. A relevant value for the diffusivity $D$ is more difficult to estimate. We quote here the value of $1.4 \times 10^{-9} \mathrm{~m}^{2} / \mathrm{s}$ used by Schwartz et al. (2001) as representative of the mixing of low-viscosity fluids. This results in a dimensionless diffusivity $d$ of 0.082 .

Figure 3 is a top view and illustrates simulation results for two sets of conditions. The first one, case a, has a high value of $\Sigma$ and a low value of $d(\Sigma=1$ and $d=0.05)$ and the second one, case $\mathbf{b}$, has a lower value of $\Sigma$ and a higher value of $d(\Sigma=0.01$ and $d=0.1$ ). For both cases, the precursor film thickness $h^{\star}$ is set to 0.04. Smaller values of the precursor film thickness would require finer meshes resulting in a steep increase in computational time. The sequence shows some of the major stages of the droplet coalescence reported in Andrieu et al. (2002): the initial contact and appearance of a neck between the two droplets, the growth of the neck at almost constant lateral extent, and the contraction 
of the droplet, possibly combined with translation, to recover a spherical cap configuration. Intuition suggests that the self-propulsion will be enhanced if $\Sigma$ is greater which essentially increases the driving mechanism (by increasing in Marangoni stresses) and $d$ is smaller which results in a longer lasting surface tension gradient since diffusion enhances the mixing of the fluids. This is precisely the trend observed in Figure 3, where the colored region indicates the footprint of the droplets system defined here as the points where the film thickness is greater than 0.05, a value slightly higher than the precursor film thickness to filter out film thickness fluctuation in the contact line region. It is clear from this picture sequence that some amount of self-propulsion is achieved for case a: the droplet advances by almost a diameter. The droplet for case $\mathbf{b}$ appears to remain fairly static, on the other hand. This is confirmed by looking at Figure 4, which gives a more quantitative picture of the coalescence and self-propulsion. For case a, the upper graph in Fig. 4, the Marangoni stresses are sufficiently strong to significantly distort the droplet from the spherical cap configuration which initiates the self-propulsion. This feature, on the other hand, is not observed for case b. The concentration contours on Figure 3 also embody useful information. Clearly, the diffusion front smears out more rapidly when the diffusivity is larger (case b) and the concentration homogenizes quicker leading to a more rapid "fuel" exhaustion as the surface tension gradient may be thought of as the fuel which propels the droplet. Also of interest is the fact that Droplet 1 mixes into Droplet 2 along the edges of the resulting droplet, a feature already observed experimentally in Sellier and Trelluyer (2009), see Figure 5. Figure 6 shows the volumetric discharge field given by eq. (4) and the contours of constant surface tension. This is again a view from above of the droplets system. The vectors show that during the initial stage of the coalescence, the flow is reminiscent of a stagnation point flow (Figures 1-3), as observed previously for free droplets coalescence in an infinite medium (Verdier and Brizard, 2002). As Droplet 1 penetrates Droplet 2, the surface tension gradient drives Droplet 1 sideways, towards the edges of the droplets system (Figures 4, 5). Finally, the droplet sets in motion and the flow field is unidirectional in the propulsion direction (Figure 6).

In order to establish more systematically the effect of $\Sigma$ and $d$ on the self-propelling behavior, a parametric study is performed. The value of $\Sigma$ is varied between 0.1 and 1 and the value of $d$ between 0.05 and 0.5 . This parameter window offers a good overview of the expected dynamics. The position of the center of gravity of the system as a function of time 

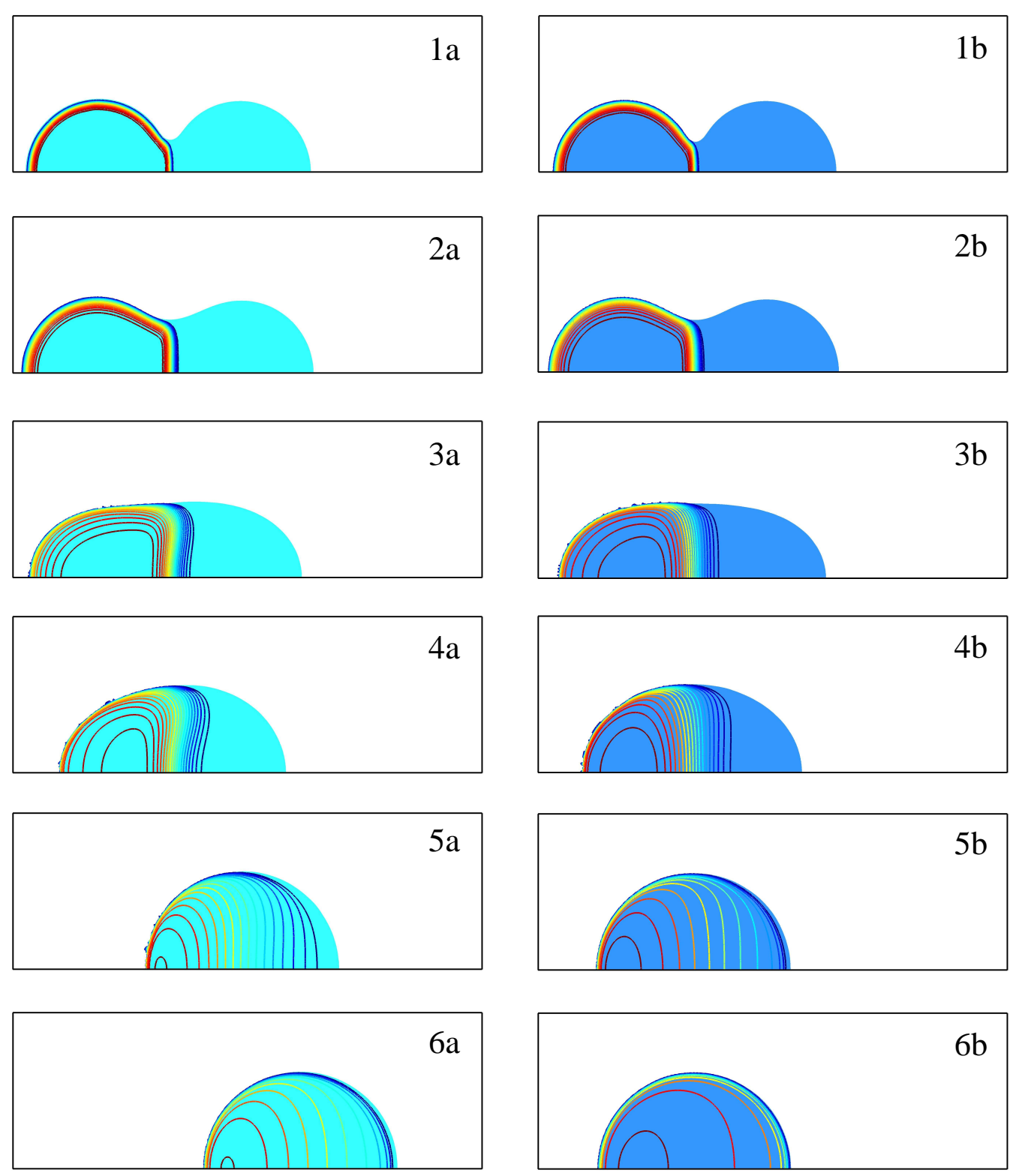

FIG. 3. Top view of the droplets system at different times. The sequence reads from left to right and top to bottom. The colored region indicates the footprint of the droplets system and the contours are lines of constant Droplet 1 fluid concentration, $c=\frac{h_{1}}{h}$. The picture number, ranging from 1 to 6 , corresponds to $t=0.036-0.1-0.464-1-4.64-10$, label a corresponds to $\Sigma=1$, $d=0.05$, and label $\mathbf{b}$ to $\Sigma=0.01, d=0.1$ 

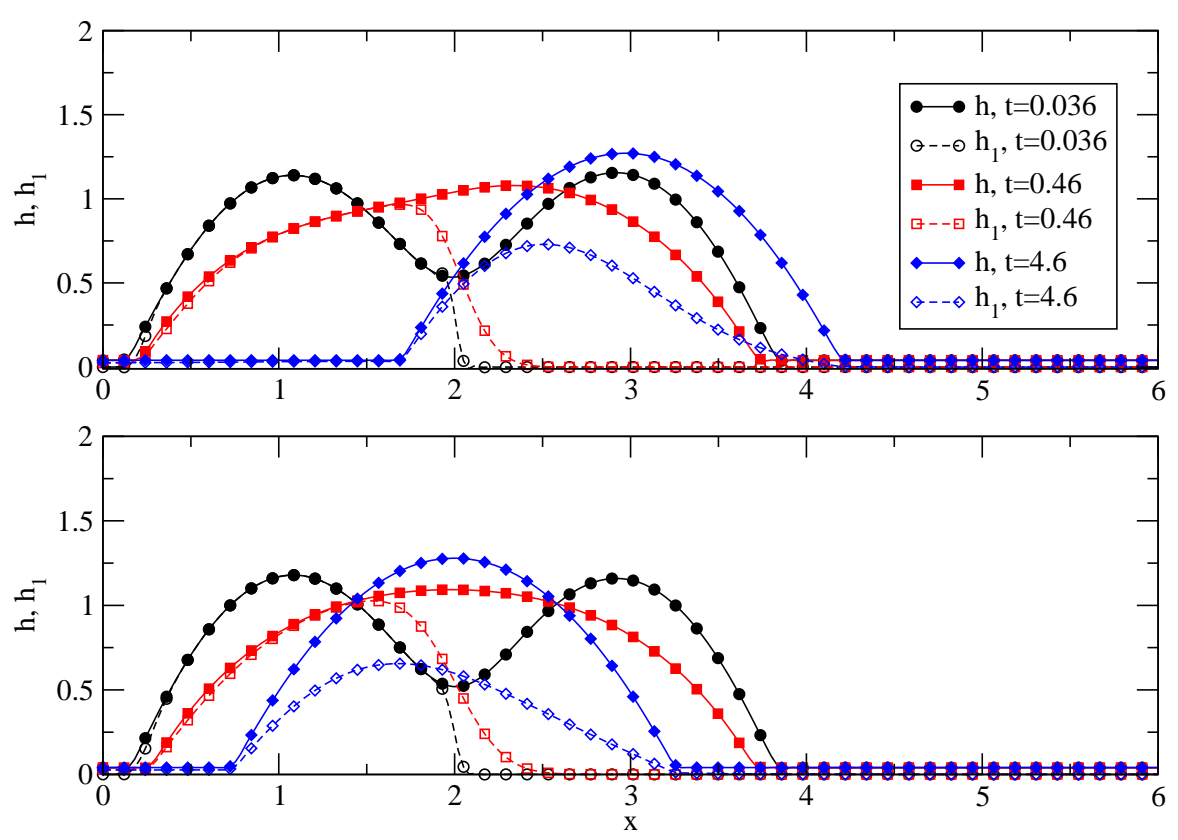

FIG. 4. Free surface and $h_{1}$ profile along $y=0$ for the same parameters as in Figure 3: case a (upper graph) and case b (lower graph).

is shown in Figure 7. It is computed according to

$$
x_{C O G}=\frac{\int_{\Omega}\left(h-h^{\star}\right) x d \omega}{\int_{\Omega}\left(h-h^{\star}\right) d \omega},
$$

where $\Omega=[0,6] \times[0,2]$ is the computational domain. All the curves in Figure 7 have a similar trend although not on the same scale. The center of gravity moves to greater values of $x$, i.e. to the right of the computational domain, as time increases. This motion is relatively fast initially, it slows down for later times, and finally the droplets system reaches an equilibrium position. At $t=30$, the droplets systems have reached (or are very near) their equilibrium positions. Figure 7 confirms that self-propulsion is maximum for larger values of $\Sigma$. The largest displacement of the droplet, $d_{\max }$, is achieved for $\Sigma=1$ and $d=0.05$ for which $d_{\max }=2.37$. For the silicone oil system discussed above, this corresponds to a total displacement of $6.2 \mathrm{~mm}$. Almost no motion is observed for $\Sigma=0.1$, the smallest tested value of the dimensionless group $\Sigma$. As previously mentioned, lower values of $d$ enhance the self-propulsion since it maintains a surface tension gradient for a longer time period. This competition between the surface tension gradient drives the system and the tendency of diffusion to steer the system towards equilibrium can be captured by the Peclet number. We express here the Peclet number, $P e$, as the ratio of the time scale for lateral diffusion, 


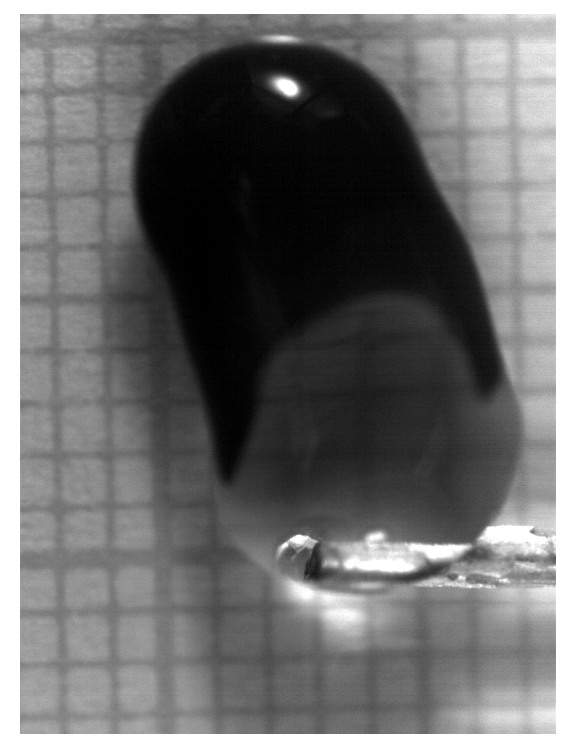

FIG. 5. Experimental observation of the mixing of two sessile droplets, source Sellier and Trelluyer (2009).
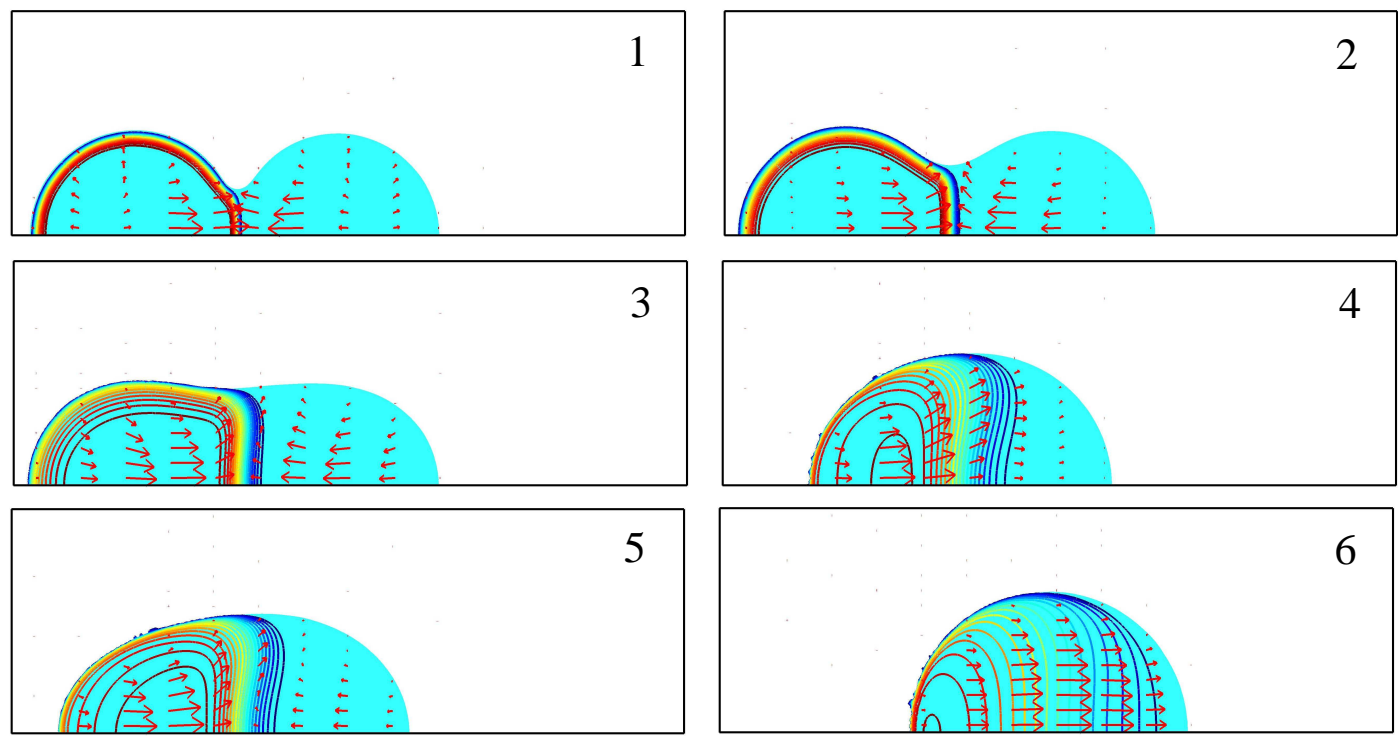

FIG. 6. Vector plot of the volumetric discharge field and contours of constant surface tension for increasing times from top-left to bottom-right. The droplets are seen from above.

$T_{D}$, to that of Marangoni flow, $T_{M}$, i.e. $P e=T_{D} / T_{M}$. The time scale for lateral diffusion is given by $T_{D}=R_{0}{ }^{2} / D$ while that of Marangoni flow is $T_{M}=R_{0} / V_{M}$ where $V_{M}$ is a typical velocity induced by surface tension gradient. For a thin layer of fluid, this velocity may 


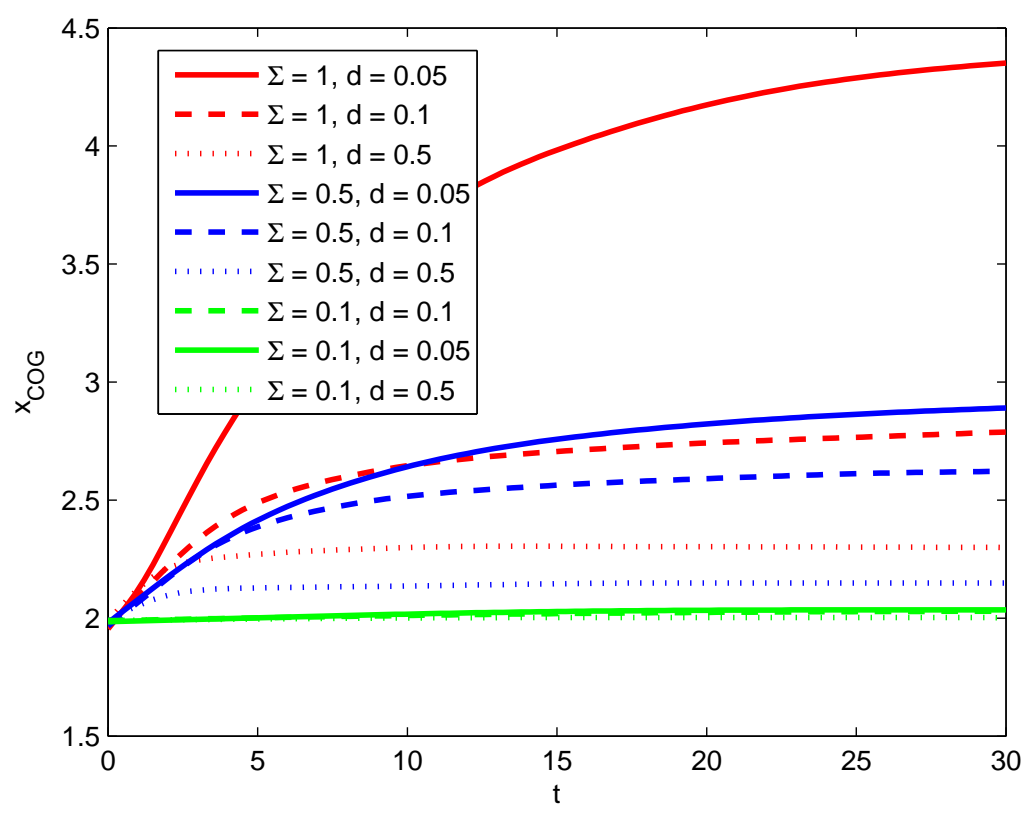

FIG. 7. Position of the center of gravity of the system as a function of time.

approximately be expressed as $H \nabla \sigma / 2 \mu$. Therefore, an order of magnitude estimate of $V_{M}$ is

$$
V_{M} \approx \frac{H_{0}}{2 \mu} \frac{\Gamma}{R_{0}}=\frac{\Gamma}{2 \mu} \epsilon
$$

After some manipulation, it can be shown that this Peclet number can be expressed in terms of the previously defined dimensionless groups as $P e=\Sigma / d$. Figure 8 shows the total traveled distance, $d_{\max }$, as a function of the Peclet number for the values of $d$ and $\Sigma$ reported in Figure 7. A line of unit slope is also plotted on this log-log graph and the good correlation between the numerical results and this line (especially for larger Peclet numbers) indicate that the total traveled distance increases linearly with the Peclet number.

The velocity of the center of gravity is reported in Figure 9. It is simply obtained by differentiating a cubic spline fitted through the points representing the position of the center of gravity as a function of time. The scientific computing program MATLAB is used for this purpose. The velocity of the center of gravity is seen to increase first as the droplet gains momentum due to the surface tension gradient. It reaches a maximum which is then followed by a decrease. The maximum speed achieved during the coalescence is again for $\Sigma=1$ and $d=0.05$. It reaches a value of 0.245 , which means that the droplets system runs over $0.245 R_{0}$ during $T_{0}$. For the silicone oil system discussed above, this would give 


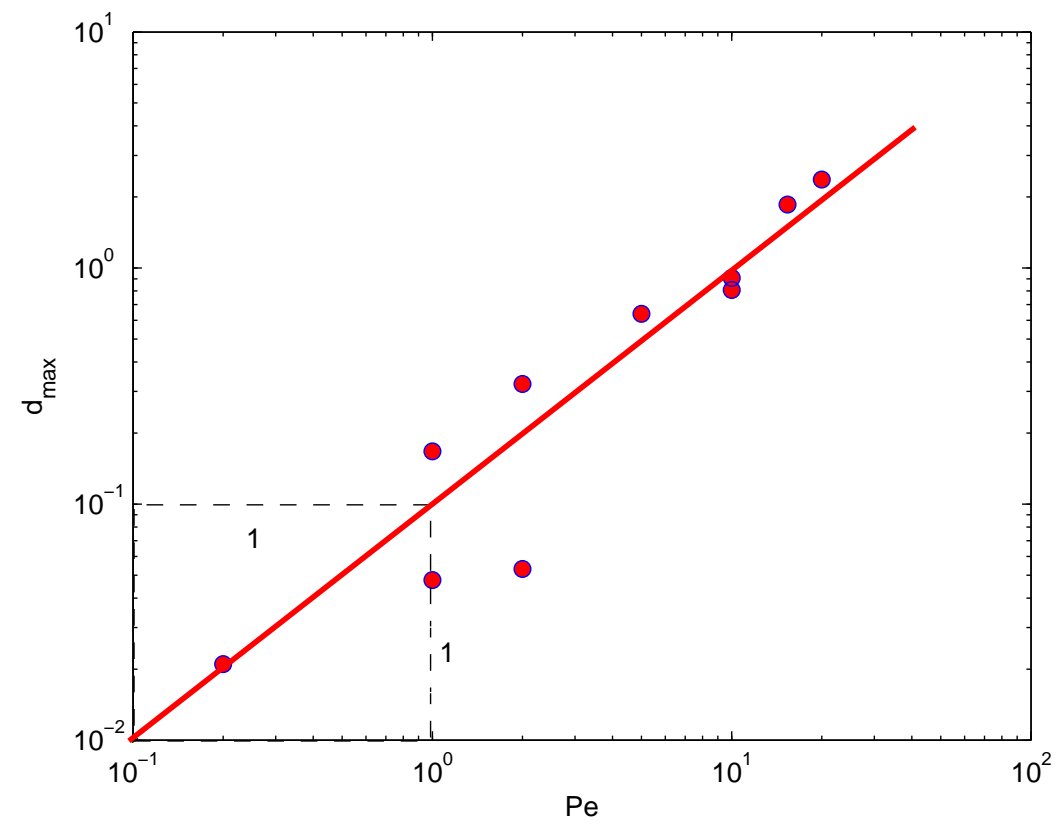

FIG. 8. Distance traveled as a function of the Peclet number.

a maximum velocity of $\approx 1.6 \mathrm{~mm} / \mathrm{s}$. This value is to be taken carefully as the contact line dynamics is known to be very dependent on the precursor film thickness with thicker films (as is the case here) tending to over-predict the spreading rate. The system velocity is almost nil when $\Sigma=0.1$. It is also worth mentioning that the peak velocity is reached for later times for smaller values of the diffusivity which reflects the fact that the surface tension gradient is stronger over a larger time interval for a small diffusivity.

\section{CONCLUDING REMARKS}

This work proposes a model for the coalescence of sessile, miscible, partially wetting droplets. The model is based on the lubrication approximation which assumes a flow with negligible inertia and a small slope of the free surface. An additional conservation equation is introduced to represent the concentration of one of the phases in the mixture. Besides the limitations of the model implicit to the lubrication approximation framework, we have further assumed that the viscosity, density, and wettability are the same for both droplets letting only surface tension differ. Bearing in mind these simplifications, the results of this work suggest that it is possible to propel droplets by using the surface tension gradient which 


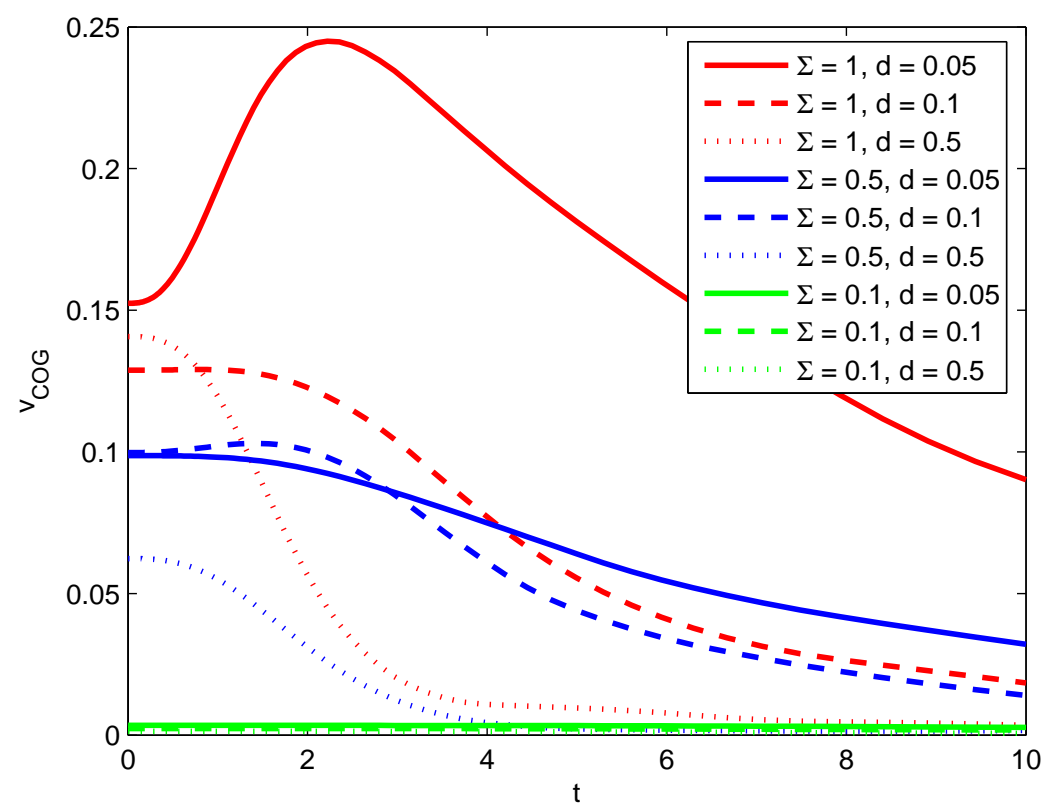

FIG. 9. Velocity of the center of gravity of the droplets system as a function of time.

arises when the two droplets merge. The governing equations derived here suggest that the strength of the self-propulsion is governed by two dimensionless numbers: $\Sigma$, a measure of the surface tension contrast and $d$, the diffusivity which gives a measure of how long it takes for the two droplets to be fully mixed. These parameters can be combined into a Peclet number and the numerical results suggest that the maximum traveled distance of the system is proportional to this number, and corresponds to a peak velocity at intermediates times. This propulsion mechanism has the advantage of being easy to implement compared to others such as thermocapillary or electrowetting which require advanced micro-fabrication techniques. It has the disadvantage, on the other hand, of not being sustainable: i.e. the propulsion will stop when mixing is complete. 


\section{ACKNOWLEDGMENT}

The authors gratefully acknowledge the support of the Dumont d'Urville New Zealand / France Science \& Technology Program.

[1] Andrieu, C., Beysens, D. A., Nikolayev, V. S. and Pomeau, Y. (2002). Coalescence of sessile drops. J. Fluid Mech. 453, 427-438.

[2] Brochard, F. (1989). Motion of droplets on solid-surfaces induced by chemical or thermalgradients. Langmuir 5, 432-438.

[3] Brzoska, J.B., Brochard-Wyart, F. and Rondelez, F. (1993). Motions of droplets on hydrophobic model surfaces induced by thermal gradients. Langmuir 9, 2220-2224.

[4] Chaudhury, M.K. and Whitesides, G.M. (1992). How to make water run uphill. Science 256, 1539-1541.

[5] Cho, S.K., Moon, H.J. and Kim, C.J. (2003). Creating, transporting, cutting, and merging liquid droplets by electrowetting-based actuation for digital microfluidic circuits. J. Microelectromech. S. 12(1), 70-80.

[6] Cho, S.K. and Moon, H. (2008). Electrowetting on dielectric (EWOD): New tool for bio/micro fluids handling. Biochip J. 2(2), 79-96.

[7] Darhuber, A.A., Valentino, J.P., Davis, J.M., Troian, S.M. and Wagner, S. (2003). Microfluidic actuation by modulation of surface stresses. Appl. Phys. Lett. 82(4), 657-659.

[8] Darhuber A.A., Valentino, J.P., Troian, S.M. and Wagner, S. (2003). Thermocapillary actuation of droplets on chemically patterned surfaces by programmable microheater arrays. J. Micro-electromech. Syst. 12, 873-879.

[9] Darhuber, A.A. and Troian, S.M. (2005). Principles of microfluidic actuation by modulation of surface stresses. Annu. Rev. Fluid Mech. 37, 425-455.

[10] Daniel, S. and Chaudhury, M.K. (2002). Rectified motion of liquid drops on gradient surfaces induced by vibration. Langmuir 18(9) 3404-3407.

[11] de Gennes, P.G. (1985). Wetting: statics and dynamics. Rev. Mod. Phys. 57, 827-963.

[12] Fair, R.B., Khlystov, A., Tailor, T.D., Ivanov, V., Evans, R.D., Griffin, P.B., Srinivasan, V., Pamula, V.K., Pollack, M.G. and Zhou, J. (2007). Chemical and biological applications of 
digital-microfluidic devices. IEEE Des. Test Comput. 24 (1), 10-24.

[13] Ford, M.L. and Nadim, A. (1994). Thermocapillary migration of an attached drop on a solid surface. Phys. Fluids 6, 3183-3285.

[14] Gaskell, P.H., Jimack, P.K., Sellier, M. and Thompson, H.M. (2004). Efficient and accurate time adaptive multigrid simulations of droplet spreading. Int. J. Num. Meth. Fluids 45, 1161.

[15] Gomba, J.M. and Homsy, G.M. (2010). Regimes of thermocapillary migration of droplets under partial wetting conditions. J. Fluid Mech. 647, 125-142.

[16] Greenspan, H.P (1978). Motion of a small viscous droplet that wets a surface. J. Fluid Mech. 84, 125-143.

[17] Howison, S.D., Moriarty, J.A., Ockendon, J.R., Terrill, E.L. and Wilson, S.K. (1997). A mathematical model for drying paint layer. J. Eng. Math. 32(4), 377-394.

[18] Renaudin, A., Tabourier, P., Zhang, V., Camart, J.C. and Druon, C. (2006). SAW nanopump for handling droplets in view of biological applications. Sens. Actuators B 133, 389-397.

[19] Ristenpart, W.D., McCalla, P.M., Roy, R.V. and Stone, H.A. (2006). Coalescence of spreading droplets on a wettable substrate. PRL 97, 064501.

[20] Schwartz, L.W., Roy, R.V., Eley, R.R. and Petrash, S. (2001). De-wetting patterns in a drying liquid film J. Colloid Interface Sci. 234, 363-374.

[21] Schwartz, L.W., Weidner, D.E. and Eley, R.R. (1995). An analysis of the effect of surfactant on the leveling behavior of a thin liquid coating layer. Langmuir 11, 3690-3693.

[22] Schwartz, L.W. and Eley, R.R. (1998). Simulation of droplet motion on low-energy and heterogeneous surfaces. J. Colloid Interface Sci. 202, 173-188.

[23] Schwartz, L.W. (1998). Hysteretic effects in droplet motion on heterogeneous substrates: direct numerical simulation. Langmuir 14, 3440-3453.

[24] Sellier, M. and Trelluyer, E. (2009). Modeling the coalescence of sessile droplets. Biomicrofluidics 3, 022412.

[25] Sellier, M. and Panda, S. (2010). Beating Capillarity in thin film flows. Int. J. Num. Meth. Fluids 63, 431-448.

[26] Smith, M.K. (1995). Thermocapillary migration of a two-dimensional liquid droplet on a solid surface. J. Fluid Mech. 294, 209-230.

[27] Stone, H.A., Stroock, A.D. and Ajdari, A. (2004). Engineering flows in small devices: Microfluidics toward a lab-on-a-chip. Annu. Rev. Fluid Mech. 36, 381-411. 
[28] Verdier, C. and Brizard, M. (2002). Understanding droplet coalescence and its use to measure interfacial tension. Rheol. Acta 41, 514-523.

[29] Yarin A.L., Liu, W. and Reneker, D.H. (2002). Motion of droplets along thin fibers with temperature gradient. J. Appl. Phys. 91, 4751-4760.

[30] Yeo, L.Y. and Chang, H.-C (2005). Static and Spontaneous Electrowetting. Mod Phys Lett B 19, 549-569.

[31] Yeo, L.Y. and Friend, J.R. (2009). Ultrafast microfluidics using surface acoustic waves. Biomicrofluidics 3, 012002. 